\title{
Conhecimento dos profissionais de enfermagem sobre sepse - estudo em um hospital universitário de Fortaleza/Ceará
}

\section{Nursing knowledge about sepsis - a study in a university hospital of Fortaleza/Ceará}

Tales Torricelli de Sousa Costa e Silva ${ }^{1}$. Jorge Luiz Nobre Rodrigues ${ }^{2}$. Germana Perdigão Amaral ${ }^{3}$. Arnaldo Aires Peixoto Júnior ${ }^{4}$.

1 Graduado em Medicina, Faculdade de Medicina Nova Esperança (FAMENE), residência em Clínica Médica, Hospital Universitário Walter Cantídio (HUWC), Universidade Federal do Ceará (UFC), Fortaleza, Ceará, Brasil. 2 Doutor em Doenças Infecciosas, Universidade Federal de São Paulo (UNIFESP). Professor do Departamento de Saúde Comunitária, Faculdade de Medicina, Universidade Federal do Ceará (UFC), Fortaleza, Ceará, Brasil. 3 Graduada em Enfermagem, Universidade Federal do Ceará (UFC), Enfermeira da Comissão de Controle de Infecção Hospitalar, Hospital Universitário Walter Cantídio (HUWC-UFC), Fortaleza, Ceará, Brasil. 4 Doutor em Farmacologia, Universidade Federal do Ceará (UFC), Professor do Departamento de Medicina Clínica, Faculdade de Medicina, Universidade Federal do Ceará (UFC), Fortaleza, Ceará, Brasil.

\section{RESUMO}

Objetivo: neste estudo procurou-se avaliar o conhecimento sobre sepse por profissionais de enfermagem do Hospital Universitário Walter Cantídio, Universidade Federal do Ceará (HUWC/UFC). Métodos: estudo transversal e exploratório. Os dados foram coletados por meio de questionários aplicados durante o turno de trabalho nas enfermarias e UTI. Resultados: 80 profissionais participaram do estudo, destes, $65 \%$ eram técnicos de enfermagem, $85,0 \%$ eram do sexo feminino, $33,75 \%$ tinham entre 30 e 40 anos de idade e $80,0 \%$ trabalhavam na enfermaria. O maior percentual (32,5\%) tinha até 5 anos de experiência de trabalho, sendo que 25 profissionais $(31,3 \%)$ possuíam pós-graduação e treinamento anterior sobre sepse foi recebido por 5,0\%. O item "conceito de sepse" e os relativos aos valores dos parâmetros dos "sinais clínicos de sepse" foram respondidos de forma correta por um percentual inferior a 50,0\% dos profissionais. Valores de "hipertermia ou hipotermia associados à sepse" foram os únicos identificados por $75,0 \%$ dos profissionais entrevistados. Os itens sobre "condutas ao se identificar sinais e sintomas de sepse" e "tempo recomendado para o início da administração do antibiótico" foram respondidos corretamente por 72,5 e 78,75\%, dos profissionais, respectivamente. Conclusão: a avaliação sobre sepse revelou a presença de lacunas do conhecimento sobre o conceito de sepse e os sinais relativos a essa condição, justificando necessidade de treinamento para profissionais de enfermagem entrevistados do Hospital Universitário Walter Cantídio-Universidade Federal do Ceará.

Palavras-chave: Síndrome de resposta inflamatória sistêmica. Sepse. Sinais e sintomas. Enfermagem.

\section{ABSTRACT}

Objective: to evaluate knowledge about sepsis by nursing professionals. Methods: transversal and exploratory study. Data were collected through questionnaires applied during work in the wards and ICU. Results: a total of 80 professionals participated in the study, $65 \%$ of which were nursing technicians. They were women $(85.0 \%)$, between 30 and 40 years old $(33.75 \%)$ and worked in the ward (80.0\%). The highest percentage (32.5\%) had up to 5 years of professional experience, a total of 25 professionals (31.3\%) had postgraduate degrees and previous training in sepsis was received by $5.0 \%$. The item "concept of sepsis" and those related to the values of the parameters of "clinical signs of sepsis" were correctly answered by a percentage lower than $50.0 \%$ of the professionals. The values of "hyperthermia or hypothermia associated with sepsis" were the only ones identified by the majority of professionals (75.0\%). The items on "conduct in the identification of signs and symptoms of sepsis" and "recommended time for the start of administration of antibiotics" were answered correctly by 72.5 and $78.75 \%$ of the professionals, respectively. Conclusion: the evaluation about sepsis revealed the presence of knowledge gaps on the concept of sepsis and the signs related to this condition among nursing professionals, justifying the need for training for these professionals.

Keywords: Systemic inflammatory response syndrome. Sepsis. Signs and symptoms. Nursing.

Autor correspondente: Tales Torricelli de Sousa Costa e Silva, Rua Capitão Francisco Pedro, 1290, Rodolfo Teófilo, Fortaleza, Ceará. CEP: 60.430370. Telefone: +55 85 99808-3798. E-mail: talestorricelli@hotmail.com

Conflito de interesses: Não há qualquer conflito de interesses por parte de qualquer um dos autores.

Recebido em: 13 Dez 2016; Revisado em: 10 Fev 2017; Aceito em: 26 Mar 2017. 


\section{INTRODUÇÃO}

A sepse é um problema de saúde mundial que vem preocupando as autoridades em saúde. A mortalidade mundial por sepse grave no ambiente hospitalar chega a 49\%. ${ }^{1}$ O Brasil apresenta a segunda maior taxa de mortalidade por sepse no mundo, com índices de aproximadamente 50 a $60 \%$ e ocupando a principal causa de óbito intra-hospitalar. ${ }^{2}$ Em uma revisão realizada no banco de dados do Núcleo de Epidemiologia Hospitalar, de 236 declarações de óbito do Hospital Universitário Walter Cantídio da Universidade Federal do Ceará (HUWC/UFC) referentes ao ano de 2013 , um percentual de $62 \%$ identificou como causa morte a sepse ou o choque séptico. ${ }^{3}$

Uma importante atitude para redução da mortalidade dos pacientes com sepse e sepse grave é a identificação rápida e precisa dos sinais e sintomas e o início precoce do tratamento adequado, como relata o protocolo da campanha de sobrevivência à sepse do Instituto Latino Americano da Sepse (ILAS). No entanto, para o diagnóstico precoce, é necessário que haja o conhecimento, por parte dos profissionais de saúde, acerca das definições de Síndrome da Resposta Inflamatória Sistêmica (SIRS), Sepse, Sepse grave e Choque séptico.

Técnicos de enfermagem e enfermeiros têm papel fundamental na identificação precoce dos sinais e sintomas de sepse e na abordagem adequada precoce ao sinalizar essa condição grave para os demais membros da equipe. Esse papel pode ser desempenhado por estes profissionais, seja na emergência, enfermaria ou Unidade de Terapia Intensiva (UTI). Isso evita a evolução dessa condição, a qual pode findar em disfunções orgânicas e em choque séptico. ${ }^{4}$

Tendo em vista o papel destes profissionais frente ao tratamento da sepse, justifica-se a aferição do conhecimento sobre a definição dessa condição, dos seus sinais e sintomas associados e da abordagem adequada.

Portanto, esse estudo tem como objetivo avaliar o conhecimento dos profissionais de enfermagem de um hospital universitário sobre o conceito de sepse, os parâmetros clínicos utilizados para identificá-la e a abordagem inicial adequada, uma vez que a sepse é a principal causa de mortalidade no HUWC/UFC.

\section{MATERIAIS E MÉTODOS}

Trata-se de um estudo transversal, exploratório, realizado por meio de um questionário direcionado a profissionais do corpo efetivo de enfermagem (enfermeiros e técnicos de enfermagem), e aplicado nas enfermarias clínicas (IA, IIA e IIB) e UTI Clínica do HUWC/UFC durante os meses de janeiro e fevereiro de 2015. A pesquisa recebeu aprovação do Comitê de Ética em Pesquisa, tendo sido registrada com o número 0180215.

A seleção da amostra foi realizada por conveniência, a partir da lista de profissionais convidados pela Comissão de Controle de Infecção Hospitalar (CCIH), para participação voluntária de um treinamento sobre protocolo de identificação e atendimento de paciente com sepse baseado no Instituto Latino-americano de Sepse (ILAS).
Os critérios utilizados para inclusão foram ser profissionais enfermeiros ou técnicos de enfermagem efetivos do HUWC, diretamente ligados à assistência aos pacientes internados no hospital durante o período de trabalho. Para evitar vieses, foram excluídos os profissionais enfermeiros ou técnicos de enfermagem que atuavam apenas em atividades administrativas ou que fizeram parte da CCIH.

Todos os profissionais que foram convidados concordaram em participar, após a leitura e assinatura do Termo de Consentimento Livre e Esclarecido. Com o intuito de aumentar a adesão, a aplicação do questionário ocorreu durante o turno de trabalho, em momento adequado para que não comprometesse o trabalho profissional e nem a avaliação.

O questionário foi elaborado pelo autor principal e a validação de conteúdo foi realizada por comitê formado por dois médicos especialistas da CCIH, designados como juízes, por meio de método qualitativo e de processo interativo entre pesquisador e membros do comitê, conforme recomendação da literatura., 5 Em seguida, este foi aplicado de acordo com horário de trabalho, turnos da manhã, tarde e noite, sendo esse composto por 12 itens objetivos do tipo múltipla escolha, com informações relativas a dados demográficos, sobre formação profissional, local de atuação e tempo de experiência profissional. Uma segunda parte do questionário listava perguntas relativas ao conceito de sepse, valores dos parâmetros clínicos utilizados para identificar essa condição, situações especiais que podem dificultar estes sinais e conduta inicial adequada ao identificar a sepse. O tempo médio de aplicação do questionário foi de 30 minutos. Tal questionário foi elaborado de acordo com conteúdo do ILAS.

As definições consideradas corretas a serem identificadas por profissionais de enfermagem foram: ${ }^{7}$

- Síndrome da Resposta Inflamatória Sistêmica (SRIS): Presença de pelo menos 2 dos seguintes itens:

a) temperatura central $>38,3{ }^{\circ} \mathrm{C}$ (hipertermia) ou $<36$ ${ }^{\circ} \mathrm{C}$ (hipotermia);

b) frequência cardíaca $>90$ bpm (taquicardia);

c) frequência respiratória $>20 \mathrm{rpm}$ (taquipneia).

- Sepse: SRIS secundária a processo infeccioso confirmado ou suspeito, sem necessidade da identificação do agente infeccioso.

- Sepse grave: presença dos critérios de sepse associada à disfunção orgânica ou sinais de hipoperfusão:

a) Oligúria: $<0,5 \mathrm{ml} / \mathrm{Kg} / \mathrm{h}$;

b) Rebaixamento do nível de consciência.

- Situações especiais:

a) Hipotensão no paciente previamente hipertenso: 
queda maior ou igual a $40 \mathrm{mmHg}$ da pressão arterial basal do paciente;

b) Ausência de sinais e sintomas de SIRS na sepse grave: a ausência dos critérios de SIRS não exclui o diagnóstico de sepse grave. Alguns pacientes, principalmente idosos e imunossuprimidos, não apresentam estes sinais.

- Abordagem inicial dos profissionais de enfermagem:

a) Conduta ao identificar sinais e sintomas de SIRS/ Sepse grave: chamar o médico.

b) Tempo para administração de antibiótico: iniciar antibioticoterapia empírica em 1 hora.

Os dados foram plotados em tabela usando o programa Excel para Mac 2011 (versão: 14.5.5 - Microsoft $^{\circledR}$ ) e expressos em valores absolutos e relativos. As variáveis trabalhadas estão descritas na Tabela 1 e Gráfico 1, respectivamente.

\section{RESULTADOS}

Um total de 80 profissionais participaram do estudo, sendo a maioria do sexo feminino. $90 \%$ dos participantes tinham idade entre 18 e 50 anos e eram formados por técnicos de enfermagem. As enfermarias foram os locais de maior frequência de trabalho (64\%), e (56,2\%) dos profissionais tinham menos de 10 anos de experiência profissional, predominando com até 5 anos trabalhando nessa área. (31\%) da amostra tinha curso de pósgraduação, sendo essa composto apenas por enfermeiros. Além disso, apenas $5 \%$ dos profissionais haviam feito algum curso sobre sepse, sendo todos também enfermeiros (Tabela 01).

O item sobre conhecimento do conceito de sepse, conforme o ILAS, foi acertado por apenas $40,0 \%$ dos profissionais, sendo estes igualmente distribuídos entre enfermeiros e técnicos de enfermagem.

Os valores de referência para detecção de hipertermia e hipotermia na sepse foi o item que obteve maior percentual de acerto $(75,0 \%)$, sendo estes valores do conhecimento de $76,9 \%$ do total para técnicos de enfermagem e de $71,4 \%$ para os enfermeiros. Os percentuais de acertos para definição de hipotensão, taquipneia, taquicardia e oligúria entre todos os profissionais entrevistados foram de: $35,0 \%, 22,5 \%, 31,25$ $\%$ e $32,5 \%$, respectivamente. Além disso, o rebaixamento do nível de consciência foi identificado como um sinal clínico associado à sepse por apenas $43,75 \%$ destes profissionais. Estes resultados estão representados no Gráfico 1.

Tabela 1. Perfil demográfico dos profissionais de enfermagem (Fortaleza, CE, 2015).

\begin{tabular}{|c|c|c|c|}
\hline & $\begin{array}{c}\text { Geral } \\
(\mathrm{N}=80)\end{array}$ & $\begin{array}{l}\text { Técnicos(as) de enfermagem } \\
\qquad(\mathrm{N}=52)\end{array}$ & Enfermeiros(as) $(\mathrm{N}=28)$ \\
\hline \multicolumn{4}{|l|}{ Sexo* } \\
\hline Feminino & $68(85,0)$ & $44(84,6)$ & $24(85,7)$ \\
\hline Masculino & $12(15)$ & $8(15,4)$ & $4(14,3)$ \\
\hline \multicolumn{4}{|l|}{ Faixa etária (anos)* } \\
\hline 18 a 29 & $21(26,25)$ & $11(21,2)$ & $10(35,7)$ \\
\hline 30 a 40 & $27(33,75)$ & $19(36,5)$ & $08(28,6)$ \\
\hline 41 a 50 & $24(30,0)$ & $16(30,8)$ & $08(28,6)$ \\
\hline acima de 50 & $08(10,0)$ & $06(11,5)$ & $02(7,1)$ \\
\hline \multicolumn{4}{|l|}{ Local de Trabalho* } \\
\hline Enfermaria & $64(80,0)$ & $43(82,6)$ & $21(75,0)$ \\
\hline UTI & $16(20,0)$ & $09(17,4)$ & $07(25,0)$ \\
\hline \multicolumn{4}{|c|}{ Tempo de trabalho (anos)* } \\
\hline 0 a 5 & $26(32,5)$ & $18(34,6)$ & $13(46,4)$ \\
\hline 6 a 10 & $19(23,75)$ & $13(25,0)$ & $06(21,4)$ \\
\hline 11 a 15 & $17(21,25)$ & $08(15,4)$ & $04(14,3)$ \\
\hline 16 a 20 & $08(10,0)$ & $04(7,7)$ & $04(14,3)$ \\
\hline acima de 20 & $10(12,5)$ & $09(17,3)$ & $01(3,6)$ \\
\hline \multicolumn{4}{|l|}{ Formação prévia* } \\
\hline pós-graduação & $25(31,3)$ & $0(0)$ & $25(89,3)$ \\
\hline treinamento em sepse & $4(5,0)$ & $0(0)$ & $40(14,3)$ \\
\hline
\end{tabular}

Nota: valores expressos em números absolutos. 
Gráfico 1. Acertos sobre sepse entre técnicos(as) de enfermagem e enfermeiros(as).

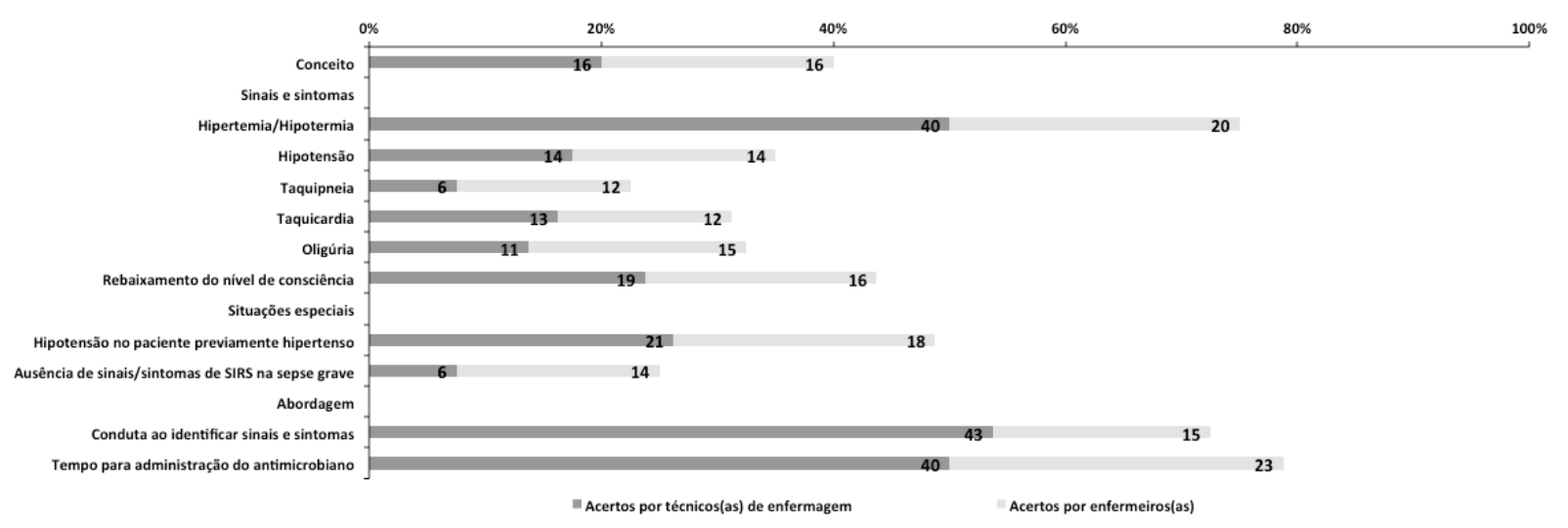

Nota: valores expressos em números absolutos.

Dentre as situações especiais na sepse, o estudo elencou os parâmetros utilizados para identificar hipotensão no paciente previamente hipertenso e identificou que apenas $48,75 \%$ dos profissionais conheciam tais parâmetros. Outra situação especial é ausência de sinais e sintomas de SIRS que pode ocorrer na sepse grave e apenas 25,0 \% dos profissionais envolvidos acertaram esse item (Gráfico 1).

Apesar da dificuldade em acertar os itens relacionados ao conceito de sepse e aos sinais e sintomas que identificam o paciente com sepse, um percentual elevado de profissionais $(72,5 \%)$ acertaram a conduta inicial ao identificar sinais e sintomas de sepse, sendo esse item acertado por $53,6 \%$ dos enfermeiros e por $82,7 \%$ dos técnicos de enfermagem, respectivamente. Um total de 78,8\% destes profissionais acertaram o valor do tempo recomendado pela literatura para administração de antimicrobiano no paciente com sepse. Estes dados também estão representados no Gráfico 1.

\section{DISCUSSÃO}

Com base na análise dos resultados verificou-se que $85 \%$ dos profissionais envolvidos eram do sexo feminino, achado que corrobora com a literatura brasileira referente ao perfil dos profissionais de enfermagem que trabalham em hospitais do Brasil. $^{8}$

O conceito de sepse, conforme o ILAS, foi acertado por apenas $40,0 \%$ dos profissionais de enfermagem, caracterizando um desconhecimento sobre a temática sepse. Sepse é a SRIS secundária a processo infeccioso confirmado ou suspeito, sem necessidade da identificação do agente infeccioso. ${ }^{7}$ Tal conceito é de suma importância para que o profissional de enfermagem reconheça e tome conduta adequada na suspeita diagnosticada. A maioria dos estudos mostra que a rápida identificação da sepse, associada à terapêutica adequada e agressiva, pode trazer resultados favoráveis para o paciente. ${ }^{9}$

Quanto aos valores de referência para detecção dos sinais de sepse, verificou-se um baixo percentual de acertos para definição de hipotensão, taquipneia, taquicardia, oligúria e rebaixamento do nível de consciência, entre todos os profissionais entrevistados.
O reconhecimento das alterações dos sinais vitais associado ao quadro de sepse são fundamentais para o diagnóstico precoce do paciente. A falta de conhecimento sobre o quadro clínico da sepse pode retardar o diagnóstico da mesma, causando prejuízos ao paciente. ${ }^{10,11}$

Dentre as situações especiais na sepse, o estudo elencou os parâmetros utilizados para identificar hipotensão no paciente previamente hipertenso e identificou que apenas $48,75 \%$ dos profissionais conheciam estes parâmetros. Tal parâmetro é de suma importância a ser reconhecido, uma vez que a disfunção cardiovascular é a manifestação mais grave do quadro séptico. ${ }^{9}$

Outra situação especial é ausência de sinais e sintomas de SIRS que pode ocorrer na sepse grave em pacientes idosos e imunossuprimidos, cenário reconhecido por apenas $25,0 \%$ dos profissionais envolvidos, caracterizando um alto percentual de desconhecimento que pode deixar passar despercebido e comprometer o prognóstico dos pacientes.

Um percentual elevado de profissionais (72,5\%) acertou a conduta inicial de chamar o médico ao identificar sinais e sintomas de sepse. Cabe ao médico confirmar a suspeita diagnosticada e solicitar exames laboratoriais, culturas de sítios pertinentes e prescrever o antibiótico adequado. ${ }^{7}$ Quanto ao tempo recomendado pela literatura para administração de antimicrobiano no paciente com sepse, 78,8\% destes profissionais acertaram, considerado então, bom resultado, já que os antibióticos administrados em até uma hora nos casos de sepse grave e choque séptico podem diminuir mortalidade. Cada hora de atraso na administração da antibioticoterapia adequada nos casos de choque séptico representa aumento significativo da mortalidade. ${ }^{12}$

Para diminuir a morbimortalidade por sepse, é importante preveni-la e, uma vez presente, é fundamental que o diagnóstico e o tratamento sejam feitos o mais rápido possível. Nesse sentido, é prioritário que a equipe de enfermagem tenha pleno conhecimento de todo o processo de identificação, cuidados e tratamento no atendimento ao paciente séptico. ${ }^{9}$

Em 2011, Aitken LM et al. realizaram um estudo baseado na avaliação das condutas recomendadas pelo protocolo 
de sepse grave e conseguiram listar 63 recomendações referentes ao cuidado de enfermagem em pacientes com sepse, incluindo medidas de prevenção e educação, prestação de contas, vigilância de infecções nosocomiais, higiene das mãos e prevenção de infecção respiratória em pacientes com cânula traqueal, infecções de catéter central e infecções do trato urinário. Segundo estes autores, o achado justifica uma necessidade urgente de maior formação nessa área do cuidado dos pacientes. ${ }^{13}$

Kent \& Fields (2012) realizaram um estudo sobre identificação de pacientes com sepse na emergência de um hospital antes e após o treinamento da equipe de enfermagem em um instrumento de rastreio para detecção da sepse severa. Estes autores identificaram um aumento significativo da detecção dessa condição após a participação destes profissionais na capacitação. ${ }^{14}$ Estes estudos prévios justificam a importância em buscarmos conhecer previamente o entendimento do corpo de enfermagem do HUWC sobre sepse.

Com objetivo de sistematizar esse conhecimento para os profissionais de enfermagem, alguns autores compilaram conhecimentos sobre a assistência da enfermagem em pacientes com sepse grave e choque séptico na emergência, baseados na atualização dos protocolos de atendimento desta condição. ${ }^{15}$

Uma iniciativa brasileira de abordagem da sepse a partir do treinamento da enfermagem foi realizada no Hospital Israelita Albert Einstein, São Paulo/SP. Os autores desse trabalho desenvolveram um programa de treinamento de uma semana chamado "Mantenha seus olhos na sepse". Este treinamento, centrado na equipe de enfermagem, tornou-se necessário à medida que as taxas de mortalidade por sepse nessa instituição mais que dobrou de 2009 para 2010, passando de 16\% para $46 \%{ }^{16}$

Em outro estudo realizado por Delaney et al. (2015), a equipe composta por 82 enfermeiros da UTI e da emergência de um hospital em Nova Iorque/EUA foi avaliada quanto ao conhecimento sobre sepse por meio, também, de um questionário com questões de múltipla escolha, autoaplicável, e identificou um conhecimento prévio satisfatório destes profissionais. Entretanto, tais autores, ainda assim, conseguiram ter algum ganho de conhecimento através de estratégias interativas de ensino. Este resultado motiva a implantação de programas de capacitação sobre sepse para equipes de enfermagem, mesmo em situações em que o conhecimento prévio é adequado. ${ }^{17}$

\section{REFERÊNCIAS}

1. Beale R, Reinhart K, Brunkhorst FM, Dobb G, Levy M, Martin G, et al. Promoting Global Research Excellence in Severe Sepsis (PROGRESS): lessons from an international sepsis registry. Infection. 2009;37(3):222-32.

2. Silva E, Pedro MA, Sogayar AC, Mohovic T, Silva CL, Janiszewski M, et al. Brazilian Sepsis Epidemiological Study (BASES study). Crit Care. 2004;8(4):R251-60.
O resultado divergente do presente estudo, quando comparado com o anterior, pode ser justificado pelo fato de que, na amostra em curso, termos avaliado todas as categorias da enfermagem e não apenas enfermeiros, e que trabalhavam não apenas em UTI, mas também em ambiente de enfermaria. Além disso, verificou-se que apenas 5\% deve ter tido algum curso de capacitação prévio que abordasse o assunto sepse.

Com o intuito de mostrar o impacto da experiência profissional prévia, capacitação prévia, nível de graduação e pós-graduação do profissional de enfermagem sobre infecções cirúrgicas e sepse, um estudo realizado na Coréia do Sul identificou uma relação inversa entre uma gradação da formação e conhecimento da equipe de enfermagem e a mortalidade em pacientes atendidos por estes profissionais, com diagnóstico de infecção e sepse. ${ }^{18}$

Tal resultado pode ter sofrido influência da formação prévia e experiência profissional na área, tendo em vista que a maioria tinha menos de 10 anos de atuação em enfermagem, uma pequena parcela $(35 \%)$ de nossa amostra tinha graduação em enfermagem e curso de pós-graduação (25\%).

As limitações do presente estudo foram o fato de este ter sido realizado em um centro único, de profissionais de enfermagem avaliados apenas por teste de múltipla escolha, em horário de trabalho. Apesar disso, identificar a conhecimento prévio da equipe de enfermagem sobre uma condição grave que tem impacto na mortalidade dos pacientes é fundamental. Até porque estes profissionais são os que identificam e que estão diretamente envolvidos em uma primeira abordagem da terapêutica da sepse. Apesar destas limitações, a importância deste estudo está no fato de ser um dos poucos com esse propósito na literatura; de ser em um hospital de ensino o qual tem como papel fundamental a formação de outros profissionais; e de servir como estímulo para a replicação em outros serviços e centros.

\section{CONCLUSÃO}

Por meio deste estudo, verificou-se um desconhecimento pela maior parte dos profissionais de enfermagem envolvidos, sobre sinais de sepse e sepse grave e no reconhecimento de hipotensão no paciente previamente hipertenso com sepse, bem como identificar sepse em idosos e imunosuprimidos. Tais fatos, alertam aos gestores do HUWC/UFC a planejar medidas de melhorias como: cursos de capacitação sobre sepse e medidas de prevenção e educação em saúde, com intuito de melhor cuidar dos pacientes e de diminuir a morbimortalidade por sepse.

3. Hospital Universitário Walter Cantídio. Núcleo de Epidemiologia Hospitalar. Arquivo de dados digitalizados das Declarações de Óbito. Fortaleza: HUWC; 2014. (Acesso em junho de 2014).

4. Sogayar AM, Machado FR, Rea-Neto A, Dornas A, Grion CM, Lobo SM, et al. A multicentre, prospective study to evaluate costs of septic patients in Brazilian intensive care units. Pharmacoeconomics. 2008;26(5):425-34. 
5. Grant JS, Davis LL. Selection and use of content experts for instrument development. Res Nurs Health. 1997;20(3):269-74.

6. Berk RA. Importance of expert judgment in content-related validity evidence. West J Nurs Res. 1990;12(5):659-71.

7. Ministério da Saúde; Sociedade Beneficente Israelita Brasileira Hospital Albert Einstein; Instituto Latino Americano de Sepse. Controlando a infecção, sobrevivendo a Sepse: manual de abordagem inicial da sepse grave e choque séptico. 1. ed. São Paulo; 2012.

8. Fronza FC, Teixeira LR. Perfil dos profissionais de saúde que trabalham em hospitais: relação entre sintomas musculoesqueléticos e qualidade de vida. Revista Brasileira de Ciencias da Saúde. 2010;8(24):53-61.

9. Conselho Regional de Enfermagem de São Paulo. Sepse, um problema de saúde pública: a atuação e colaboração da enfermagem na rápida identificação e tratamento da doença. São Paulo: COREN; 2016.

10. Westephal GA, Silva E, Salomão R, Bernardo WM, Machado FR. Diretrizes para o tratamento da sepse grave/choque séptico-ressuscitação hemodinâmica. Rev Bras Ter Intensiva. 2011;23(1):13-23.

11. Almeida AP, Belchior PK, Lima MG, Souza LP. Conhecimento do profissional de enfermagem a respeito da Sepse. Braz J Surg Clin Res. 2013;4(4):5-10.
12. Kumar A, Roberts D, Wood KE, Light B, Parrillo JE, Sharma $\mathrm{S}$, et al. Duration of hypotension before initiation of effective antimicrobial therapy is the critical determinant of survival in human septic shock. Crit Care Med. 2006;34(6):1589-96.

13. Aitken LM, Williams G, Harvey M, Blot S, Kleinpell R, Labeau $\mathrm{S}$, et al. Nursing considerations to complement the Surviving Sepsis Campaign guidelines. Crit Care Med. 2011;39(7):1800-18.

14. Kent N, Fields W. Early recognition of sepsis in the emergency department: an evidence-based project. J Emerg Nurs. 2012;38(2):139-43.

15. Rosini JM, Srivastava N. The 2012 guidelines for severe sepsis and septic shock: an update for emergency nursing. J Emerg Nurs. 2013;39(6):652-6.

16. Shiramizo SC, Assunção MS, Pardini A, Santos AM, Capone A Neto, Silva E. Keep your eyes on sepsis. Crit Care Nurse. 2012;32(6):74-5.

17. Delaney MM, Friedman MI, Dolansky MA, Fitzpatrick JJ. Impact of a sepsis educational program on nurse competence. J Contin Educ Nurs. 2015;46(4):179-86.

18. Kim Y, Cho SH, June KJ, Shin SA, Kim J. [Effects of hospital nurse staffing on in-hospital mortality, pneumonia, sepsis, and urinary tract infection in surgical patients]. J Korean Acad Nurs. 2012;42(5):719-29. Korean.

\section{Como citar:}

Costa e Silva TT, Rodrigues JL, Amaral GP, Peixoto AA Júnior. Conhecimento dos profissionais de enfermagem sobre sepse - estudo em um hospital universitário de Fortaleza/Ceará. Rev Med UFC. 2017 set-dez;57(3):24-29. 